\title{
High quality multi-frequency seismic profiles obtained during KY06-01 cruise in the Sagami Bay, Japan
}

\author{
Masataka Kinoshita $^{1}$, Mikiya Yamashita ${ }^{1}$, Tadashi Okano², Toru Nakasone ${ }^{2}$, Fujio Yamamoto \\ and Satoshi Shimizu ${ }^{3}$
}

\begin{abstract}
In February 2006, intensive seismic survey was carried out in Sagami Bay area, central Japan, during the KY06-01 cruise using R/V Kaiyo of JAMSTEC (Japan Agency for Marine-Earth Science and Technology). This cruise follows the previous KY05-06 cruise survey, in which we conducted single-channel seismic survey in the same area. Used tools are a 18 channel streamer with two 355 in $^{3}$ GI guns, and a single-channel streamer with a cluster gun. We obtained 21 profiles with GIguns in the eastern Sagami Bay, 20 profiles with GI-gun in the western Sagami Bay and 20 profiles with cluster gun near Hatsushima cold seep communities in the western Sagami Bay. Very clear acoustic images were captured with different frequencies on the same lines. We present here the seismic profiles obtained during the KY06-01 cruise.
\end{abstract}

Keywords: Sagami bay, MCS Survey, Hatsushima cold seep community, Seismic swarm activity

\section{Introduction}

The Sagami Bay, central Japan, is known to be in the most complicated tectonic area in the world. Subduction of Philippine Sea plate beneath mainland Honshu is significantly obstructed by the collision of Izu Peninsula, and many active faults are identified (Research Group of Active Faults of Japan, 1991). The volcanic front of Izu-Bonin Arc extends northward from Izu-Oshima through Higashi-Izu monogenetic volcanoes. Active cold seep biological communities are distributed along the escarpment of the eastern rim of the Izu Peninsula. Extensive earthquake swarms repeatedly occur in the eastern Izu region (Okada et al., 2000). Recently, largescale seismic reflection surveys are conducted by the Special Project for Earthquake Disaster Mitigation in Urban Areas in onshore. Sato et al. (2005) identified the megathrust fault from these deep seismic reflection profiles, and showed the upper surface of the Philippine sea plate that is shallower than previous estimates based on the distribution of seismicity. However, their result is not clear the shallow crustal structure under the Sagami Bay. Therefore, it is important for implication of whole tectonics to obtain the offshore information.

Intensive geological, geophysical, geochemical and biological surveys have been carried out in this area.
Seismic reflection surveys have been conducted (e.g. Iwabuchi et al., 1990; Kato et al., 1993). They show that the basement of Izu Peninsula tilts down north-eastward and that overlying sediment on the Sagami Trough is $4 \mathrm{~km}$ thick (Kato, 1999). They show that the basement of Izu Peninsula tilts down north-eastward and that overlying sediment on the Sagami Trough is $4 \mathrm{~km}$ thick (Kato, 1999).

During the KY05-06 cruise, we already conducted a single-channel seismic (SCS) survey in the overall Sagami Bay area in order to obtain a series of high-quality, dense seismic profiles (Kinoshita et al., 2005). This study, as a continuation of KY05-06 survey, attempts to obtain both the shallower and deeper extrapolation of some seismic lines using SCS with a cluster gun and Multi-Channel Seismic (MCS) survey with two GI guns, respectively.

This report provides preliminary profiles obtained during this cruise.

\section{Data acquisition}

\subsection{Eastern Sagami Bay Survey (Leg1)}

MCS survey was carried out from January 30, 2006 to Feburuary 5, 2006, using JAMSTEC R/V Kaiyo with a total length of approx. $426 \mathrm{~km}$ (Fig. 1). Activity ship

\footnotetext{
1 IFREE, JAMSTEC

2 CDEX, JAMSTEC

3 Nippon Marine Enterprize, Co. Ltd.
} 
$\log$ during Leg1 is indicated in Table 1. The GI gun system was used, in the eastern part of Sagami Bay area. Survey line information is summarized in Table 2.

\subsection{Western Sagami Bay (Hatsushima) Survey (Leg2)}

MCS and SCS survey was carried out from Feb. 5 to 11, 2006, onboard JAMSTEC R/V Kaiyo with a total length of approx. $274 \mathrm{~km}$ (MCS) in Fig. 1 and $56 \mathrm{~km}$ (SCS) in Fig.2. Activity ship log during Leg2 is shown in Table 3. A densely- gridded SCS survey during Leg2 was carried out centered at the Hatsushima cold seep site with the line spacing $100 \mathrm{~m}$. In order to obtain shallow structure beneath the seafloor, we first deployed two GI gun system (105 in3, steaming at 5 knots, $25 \mathrm{~m}$ shot interval, 50m streamer length) as well as Leg1 survey. After that CDEX's cluster gun system was used to obtain shallower images at some lines $\left(20 \mathrm{in}^{3}\right.$, steaming at 3 knots, $3 \mathrm{sec}$. shot interval, 20m streamer length) for SCS survey.

\section{MCS, SCS system and processing summary 3.1 MCS /twin GI gun system}

The GI gun system was effective to obtain acoustic images down to $1 \mathrm{sec}$ (TWT).

\section{GI gun}

The GI gun was used as a source, and the towing depth, source pressure etc are described below.
Total volume: $2 \times 355 \mathrm{in}^{3}$

(Generator $250 \mathrm{in}^{3} /$ Injector $105 \mathrm{in}^{3}$ )

Towing depth: $5 \mathrm{~m}$

Source pressure: 2,000 psi (136 atm.)

Pop interval: $25 \mathrm{~m}$

\section{Streamer}

Stealtharray streamer cable was used for the survey.

Specifications:

Active section length: $450 \mathrm{~m}$

Hydrophone group interval: 25m

Type of hydrophone: ITI Piezo polymer type

Hydrophone output: $-197.5+/-2 \mathrm{~dB}$, re $1 \mathrm{~V} / \mathrm{uPa}$

(13.3 V/Bar)

Frequency: 3 - 3k Hz@-3dB

Lead in cable paid out: $150 \mathrm{~m}$

Receiver depth: $8.0 \mathrm{~m}$

Cable depth controller: Digicource compass bird

Model 5011

Unfortunately, 6 channels at beginning, 7 channels later, out of 18 channels were dead. And there were also 3 to 8 wild traces. Thus the quality of records did not improve very much as compared with SCS records.

\section{Recording system}

The Geometrics Stratavisor NX marine system was used for data recording. The main feature of the system

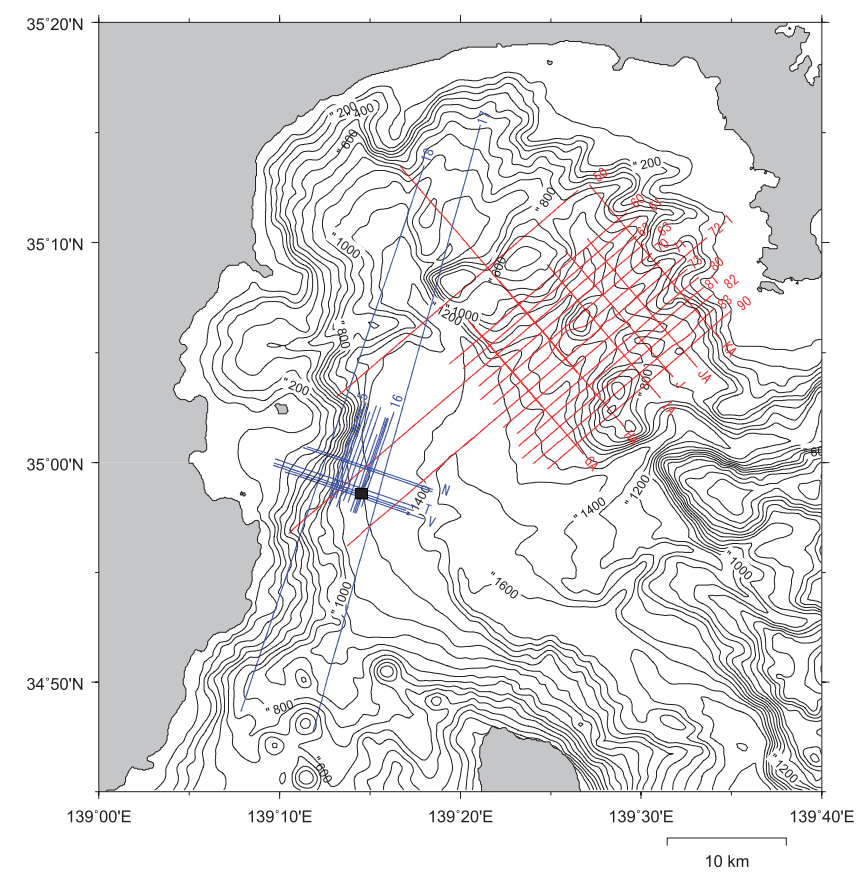

Figure 1: MCS survey line of KY06-01 cruise. Red lines show the Leg1 MCS survey. Blue lines show the Leg2 MCS survey. The square indicates the area for intensive survey (SCS) off Hatsushima. 
and parameters are:

Type of system: Stratavisor NX marine system Recording format: SEG-D 80584 byte Floating point

Recording length: $7.0 \mathrm{sec}$

Sample rate: $2 \mathrm{msec}$

High cut filter: $206 \mathrm{~Hz}$ Anti aliasing Filter

Recording media: HD/ MO

\section{Navigation}

DGPS (Sky Fix) system was used for navigation.

\subsection{Cluster gun system with SCS}

The cluster gun system is effective to obtain images for the shallower part, down to a few hundred meters.

Specifications:
(1) Manufacturer:
Bolt Technology
(2) Type of airgun: 2800LL-X Cluster
(3) Volume:
20 cuin $x 2$
(4) Air pressure:
$2000 \mathrm{psi}$
(5) Source Depth:
$1.3 \mathrm{~m}$
(6) Peak-peak:
7 bar-m
(7) Primary to bubble:
43.8
(8) Dominant frequency $(-6 \mathrm{~dB})$ :
$50 \mathrm{~Hz}-530 \mathrm{~Hz}$
(9) Depth sensor:
N/A
(10) Gun Controller:
GCS90

\subsection{Data Processing}

(1) MCS Data Processing

We used a PC-based seismic processing software "SPW" for onboard seismic QC processing and pre-processing for the later processing work. For the QC of navigation data, we used another processing software "Seispos", by which data were filtered and edited. Seismic data processing was carried out using Promax 2D (Leg1) and FOCUS (Leg2) based on the pre-processing data prepared with SPW.

We then applied band pass filter, deconvolution, Normal Moveout (NMO) correction and CMP stack in rather details. Due to short source-receiver offset and especially defective channels for MCS survey, it was not possible to conduct velocity analysis. So, 1500 $\mathrm{m} / \mathrm{sec}$ constant velocity was applied to CMP sorted data when NMO correction was applied. At the same time, velocity functions derived from an existent seismic line were applied to the newly collected seismic sections. However, no significant difference was identified between them.

\section{(2) SCS Data Processing}

SCS reflection profiles were processed for the purpose of quality control. The data processing for the overall Sagami Bay profiles contains noisy-trace editing, 14-180 Hz band-pass filtering and time migration $(\mathrm{Vp}=1500 \mathrm{~m} / \mathrm{s})$. The data processing of Hatsushima site profiles contains noisy-trace editing, 14-180 Hz (GI gun), 45-500 Hz (Cluster gun) band-pass filtering and time migration $(\mathrm{Vp}=1500 \mathrm{~m} / \mathrm{s})$.

\section{Outline of obtained data}

Approximately, $426 \mathrm{~km}$ (Leg1), $274 \mathrm{~km}$ (Leg2) of MCS and $56 \mathrm{~km}$ of SCS data were recorded for the overall Sagami Bay survey. The attached MCS images (Fig. 4) were displayed through SPW software, and bulk

Table 1: MCS line information of Leg1 for the eastern Sagami Bay survey.

\begin{tabular}{l||l}
\hline Date & Remarks \\
\hline \hline \multirow{2}{*}{ January 30 } & $\begin{array}{l}\text { Departure from Yamashita port, Yokohama } \\
\text { Transit to eastern part of Sagami-bay } \\
\text { Inspection for fish net etc } \\
\text { Adjustment of investment equipments and start airguns shooting }\end{array}$ \\
\hline \multirow{2}{*}{ January 31 } & $\begin{array}{l}\text { Continue survey } \\
\text { Recovery airguns and streamer due to bad weather }\end{array}$ \\
\hline \multirow{2}{*}{ February 1 } & $\begin{array}{l}\text { Deploy airguns and streamer } \\
\text { Restart survey } \\
\text { Recovery airguns and streamer due to Streamer's bird trouble }\end{array}$ \\
\hline \multirow{2}{*}{ February 2 } & $\begin{array}{l}\text { Deploy airguns and streamer } \\
\text { Restart survey }\end{array}$ \\
\hline \multirow{2}{*}{ February 3 } & $\begin{array}{l}\text { Continue survey } \\
\text { Recovery airguns and streamer due to bad weather }\end{array}$ \\
\hline \multirow{2}{*}{ February 4 } & $\begin{array}{l}\text { Deploy airguns and streamer } \\
\text { Restart survey }\end{array}$ \\
\hline \multirow{2}{*}{ February 5 } & $\begin{array}{l}\text { Continue survey } \\
\text { Recovery airguns and streamer } \\
\text { Transit to Ito port } \\
\text { Arrival at Ito port, end of leg1 }\end{array}$ \\
\hline
\end{tabular}


static shift was applied to the field record to compensate the recording system delay of $-35 \mathrm{msec}$ and to correct for the gun and cable depth to sea level $(9 \mathrm{msec})$.

In the western Sagami Bay off Hatsushima, we traced the same track lines using MCS and twin GI gun system as those obtained with SCS. The penetration depth did not increase very much as compared with previous SCS lines, but the overall quality improved.

Gridded survey was carried out around the possible diapir area located to the southeast of Hatsushima cold seep site (Fig. 2) (Kinoshita et al., 2006), using the single-channel and the cluster-gun system. Line spacing was 0.05 miles, and we obtained clear images of multiple diapir-like structures in this area. Details will be shown elsewhere.

We obtained two long MCS lines (Line 17 and 18) along the eastern edge of Izu Peninsula (Fig. 1), from the west of Oshima, across the Eastern Izu earthquake swarm epicentral area, and to the Manazuru Knoll (Yamashita et al., 2006). Line 17 is located in the southeastern end of tensile fault which estimated by Okada et al.(2000). We obtain tectonic and volcanic features were successfully acquired. In the northern end of Line 17, the velocity structure is obtained by Okino et al.(1994). From their results, the velocity perturbation of shallow crustal structure along this line is small. Tectonic and volcanic features were successfully acquired.

All profiles shown in this report is preliminary. Final interpretation will be reported afterwards.

\section{Acknowledgement}

We thank Captain Hitoshi Tanaka, crew and technical staffs of R/V Kaiyo, for their kind and thoughtful support during SCS survey. This survey was achieved under IFREE, CDEX of JAMSTEC.

\section{References}

1) Iwabuchi, Y., Kato, Y., Hashimoto, F., Kondo, T. and Shinbayashi, K., 1990. Multi-channel seismic reflection survey of the Sagami Bay. J. Jpn. Soc. Mar. Surv. Tech., 3, 39-51 (in Japanese).

2) Kato, S., 1999. Submarine topography and geological structure of Sagami Bay. The Quaternary Research, 38, 469-477 (in Japanese with English abstract).

3) Kato, S., Y. Iwabuchi, A. Asada, Y. Kato, S. Kikuchi, S. Kokuta, K. Kusunoki, and K. Watanabe, 1993, Crustal structure and tectonic landform of Sagami Bay, J. Geography, 102, 399-406 (in Japanese).

4) Kinoshita, M., Yamashita, M., Okano, T., Kasaya, T., Machiyama, H., and Mitsuzawa, K., 2006, Possible active structure in the western Sagami Bay detected by dense single-channel seismic survey, Japan Geoscience Union Meeting 2006, J161-027 (in Japanese).

5) Kinoshita, M., Yamashita, M., Okano, T., Shimizu, S., Hashimoto, Y., Kasaya, T. and KY05-06 Leg2 Shipboard Scientific Party, 2005. High density single-channel seismic profiles obtained during KY05-06 cruise in the Sagami Bay, Japan, JAMSTEC Rep. Res. Dev., 2, 41-56.

6) Okada, Y., E. Yamamoto, and T. Ohkubo, 2000, Coswarm and preswarm crustal deformation in the eastern Izu Peninsula, central Japan, J. Geophys. Res., 105, 681692.

7) Okino, K., Nishizawa, A. and Asada, A., 1994. Crustal structure survey in the northwestern part of the Sagami Trough. Rep. Hydrographic Res., 30, 383-393 (in Japanese with English abstract).

8) Sato, H., Hirata, N., Koketsu, K., Okaya, D., Abe, S., Kobayashi, R. Matsubara, M., Iwasaki, T., Ito, T., Ikawa, T., Kawanaka, T., Kasahara, K. and Harder S., 2005. Earthquake source fault beneath Tokyo. Science, 309, 462-464.

9) Research Group of Active Faults of Japan, 1991, Active Faults in Japan : Sheet Maps and Inventories (revised edition), Tokyo Univ. Press, B5, pp424-425.

10) Yamashita, M., Kinoshita, M., Okano, T., Takahashi, N., and Kaneda, Y., 2006, Shallow crustal structure in the Sagami Bay obtained by multi-channel seismic reflection survey, Japan Geoscience Union Meeting 2006, S118P019. 
Table 2: Activity ship log during KY06-01 cruise.

\begin{tabular}{|c|c|c|c|c|c|}
\hline Line Name & Starting Point & Ending Point & Starting Time & SP number & Line Length $(\mathrm{km})$ \\
\hline SG06-KY-90 & $\begin{array}{c}34^{\circ} 59^{\prime} 44.74 ” \mathrm{~N} \\
139^{\circ} 24^{\prime} 52.518^{\prime \prime} \mathrm{E}\end{array}$ & $\begin{array}{l}35^{\circ} 06^{\prime} 55.094^{\prime} \mathrm{N} \\
139^{\circ} 35^{\prime} 00.316^{\prime \prime} \mathrm{E}\end{array}$ & $\begin{array}{c}2006-01 / 30 \\
9: 21: 52 \\
\end{array}$ & $1002-1815$ & 20.35 \\
\hline SG06-KY-83 & $\begin{array}{c}35^{\circ} 06^{\prime} 59.00^{\prime \prime} \mathrm{N} \\
139^{\circ} 34^{\prime} 00.365^{\prime \prime} \mathrm{E}\end{array}$ & $\begin{array}{l}34^{\circ} 59^{\prime} 56.015^{\prime} \mathrm{N} \\
139^{\circ} 24^{\prime} 00.465^{\prime \prime} \mathrm{E}\end{array}$ & $\begin{array}{c}2006-01 / 30 \\
12: 28: 45\end{array}$ & $1111-1912$ & 20.05 \\
\hline SG06-KY-82 & $\begin{array}{l}35^{\circ} 00^{\prime} 18.347^{\prime \prime} \mathrm{N} \\
139^{\circ} 23^{\prime} 35.024^{\prime} \mathrm{E}\end{array}$ & $\begin{array}{l}35^{\circ} 07^{\prime} 40.726^{\prime \prime} \mathrm{N} \\
139^{\circ} 34^{\prime} 00.264^{\prime \prime} \mathrm{E}\end{array}$ & $\begin{array}{c}2006-01 / 30 \\
15: 29: 28 \\
\end{array}$ & $1538-2374$ & 20.925 \\
\hline SG06-KY-81 & $\begin{array}{l}35^{\circ} 07^{\prime} 51.429 ” \mathrm{~N} \\
139^{\circ} 33^{\prime} 16.984 ” \mathrm{E}\end{array}$ & $\begin{array}{l}35^{\circ} 00^{\prime} 43.484^{\prime \prime} \mathrm{N} \\
139^{\circ} 23^{\prime} 10.460^{\prime \prime} \mathrm{E}\end{array}$ & $\begin{array}{c}2006-01 / 30 \\
18: 34: 36\end{array}$ & $1102-1912$ & 20.275 \\
\hline SG06-KY-80 & $\begin{array}{l}35^{\circ} 01 ' 20.4022^{\prime} \mathrm{N} \\
139^{\circ} 23^{\prime} 10.4433^{\prime \prime} \mathrm{E}\end{array}$ & $\begin{array}{l}35^{\circ} 08^{\prime} 27.784^{\prime \prime} \mathrm{N} \\
139^{\circ} 33^{\prime} 13.713^{\prime \prime} \mathrm{E}\end{array}$ & $\begin{array}{c}2006-01 / 30 \\
21: 57: 57 \\
\end{array}$ & $1002-1809$ & 20.20 \\
\hline SG06-KY-73 & $\begin{array}{l}35^{\circ} 08^{\prime} 36.528^{\prime \prime} \mathrm{N} \\
139^{\circ} 32^{\prime} 31.719^{\prime \prime} \mathrm{E}\end{array}$ & $\begin{array}{l}35^{\circ} 01 ' 34.419^{\circ} \mathrm{N} \\
139^{\circ} 22^{\prime} 20.693 ” \mathrm{E}\end{array}$ & $\begin{array}{c}2006-01 / 31 \\
1: 00: 35 \\
\end{array}$ & $1112-1909$ & 19.95 \\
\hline SG06-KY-72 & $\begin{array}{c}34^{\circ} 56^{\prime} 14.099^{\prime \prime} \mathrm{N} \\
139^{\circ} 13^{\prime} 46.717^{\prime \prime} \mathrm{E}\end{array}$ & $\begin{array}{l}35^{\circ} 01^{\prime} 08.608^{\prime \prime} \mathrm{N} \\
139^{\circ} 20^{\prime} 44.390^{\prime \prime} \mathrm{E}\end{array}$ & $\begin{array}{c}2006-01 / 31 \\
6: 00: 43\end{array}$ & $1002-1560$ & 13.975 \\
\hline SG06-KY-72_1 & $\begin{array}{l}35^{\circ} 00^{\prime} 47.490{ }^{\prime} \mathrm{N} \\
139^{\circ} 20^{\prime} 14.413^{\prime \prime} \mathrm{E}\end{array}$ & $\begin{array}{l}35^{\circ} 10^{\prime} 14.799 ” \mathrm{~N} \\
139^{\circ} 33^{\prime} 38.972^{\prime \prime} \mathrm{E}\end{array}$ & $\begin{array}{c}2006-02 / 01 \\
0: 28: 52\end{array}$ & $1520-2594$ & 26.875 \\
\hline SG06-KY-71 & $\begin{array}{c}35^{\circ} 10^{\prime} 20.291 ” \mathrm{~N} \\
139^{\circ} 32^{\prime} 48.628^{\prime \prime} \mathrm{E}\end{array}$ & $\begin{array}{l}35^{\circ} 08^{\prime} 16.943 ” \mathrm{~N} \\
139^{\circ} 29^{\prime} 47.744^{\prime \prime} \mathrm{E}\end{array}$ & $\begin{array}{c}2006-02 / 01 \\
4: 20: 15\end{array}$ & $1004-1242$ & 59.75 \\
\hline SG06-KY-71_R & $\begin{array}{l}35^{\circ} 02^{\prime} 40.588^{\prime \prime} \mathrm{N} \\
139^{\circ} 21^{\prime} 51.790 ” \mathrm{E}\end{array}$ & $\begin{array}{l}35^{\circ} 09^{\prime} 09.093 ” \mathrm{~N} \\
139^{\circ} 31^{\prime} 04.765^{\prime} \mathrm{E}\end{array}$ & $\begin{array}{c}2006-02 / 02 \\
0: 26: 04 \\
\end{array}$ & $1002-1739$ & 18.45 \\
\hline SG06-KY-70 & $\begin{array}{c}35^{\circ} 09^{\prime} 26.669^{\prime} \mathrm{N} \\
139^{\circ} 30^{\prime} 29.224 ” \mathrm{E}\end{array}$ & $\begin{array}{l}35^{\circ} 02^{\prime} 50.128^{\prime \prime} \mathrm{N} \\
139^{\circ} 21^{\prime} 04.646 ” \mathrm{E}\end{array}$ & $\begin{array}{c}2006-02 / 02 \\
3: 11: 21\end{array}$ & $1157-1907$ & 18.775 \\
\hline SG06-KY-63 & $\begin{array}{l}35^{\circ} 03^{\prime} 30.203^{\prime} \mathrm{N} \\
139^{\circ} 21^{\prime} 03.492^{\prime \prime} \mathrm{E}\end{array}$ & $\begin{array}{l}35^{\circ} 09^{\prime} 58.558^{\prime \prime} \mathrm{N} \\
139^{\circ} 30^{\prime} 11.472^{\prime \prime} \mathrm{E}\end{array}$ & $\begin{array}{c}2006-02 / 02 \\
5: 50: 46 \\
\end{array}$ & $1002-1735$ & 18.35 \\
\hline SG06-KY-62 & $\begin{array}{c}35^{\circ} 10^{\prime} 09.9677^{\prime} \mathrm{N} \\
139^{\circ} 29^{\prime} 32.657^{\prime \prime} \mathrm{E}\end{array}$ & $\begin{array}{l}34^{\circ} 56^{\prime} 44.723^{\prime \prime} \mathrm{N} \\
139^{\circ} 10^{\prime} 27.863^{\prime \prime} \mathrm{E}\end{array}$ & $\begin{array}{l}2006-02 / 02 \\
8: 28: 41\end{array}$ & $1162-2689$ & 38.20 \\
\hline SG06-KY-61 & $\begin{array}{l}35^{\circ} 04^{\prime} 18.117^{\prime \prime} \mathrm{N} \\
139^{\circ} 20^{\prime} 11.254^{\prime \prime} \mathrm{E}\end{array}$ & $\begin{array}{l}35^{\circ} 11^{\prime} 15.039^{\prime \prime} \mathrm{N} \\
139^{\circ} 29^{\prime} 58.496^{\prime \prime} \mathrm{E}\end{array}$ & $\begin{array}{c}2006-02 / 02 \\
19: 06: 01 \\
\end{array}$ & $1002-1788$ & 19.675 \\
\hline SG06-KY-60 & $\begin{array}{c}35^{\circ} 11^{\prime} 18.0044^{\prime} \mathrm{N} \\
139^{\circ} 29^{\prime} 06.478^{\prime \prime} \mathrm{E}\end{array}$ & $\begin{array}{l}35^{\circ} 04^{\prime} 27.614^{\prime \prime} \mathrm{N} \\
139^{\circ} 19^{\prime} 21.505^{\prime \prime} \mathrm{E}\end{array}$ & $\begin{array}{c}2006-02 / 02 \\
22: 11: 44 \\
\end{array}$ & $1130-1909$ & 19.50 \\
\hline SG06-KY-GA & $\begin{array}{l}35^{\circ} 06^{\prime} 08.831^{\prime \prime} \mathrm{N} \\
139^{\circ} 20^{\prime} 44.628^{\prime \prime} \mathrm{E}\end{array}$ & $\begin{array}{l}35^{\circ} 022^{\prime} 29.972^{\prime \prime} \mathrm{N} \\
139^{\circ} 24^{\prime} 32.314^{\prime \prime} \mathrm{E}\end{array}$ & $\begin{array}{c}2006-02 / 03 \\
1: 34: 07 \\
\end{array}$ & $1003-1358$ & 89.00 \\
\hline SG06-KY-HA & $\begin{array}{l}35^{\circ} 04^{\prime} 07.306^{\prime \prime} \mathrm{N} \\
139^{\circ} 26^{\prime} 25.554^{\prime \prime} \mathrm{E}\end{array}$ & $\begin{array}{l}35^{\circ} 08^{\prime} 02.749 ” \mathrm{~N} \\
139^{\circ} 22^{\prime} 21.893^{\prime \prime} \mathrm{E}\end{array}$ & $\begin{array}{c}2006-02 / 03 \\
3: 16: 27\end{array}$ & $1223-1624$ & 10.05 \\
\hline $\begin{array}{c}\text { SG06-KY-HA } \\
\text { R } \\
\end{array}$ & $\begin{array}{c}35^{\circ} 13^{\prime} 22.817^{\prime \prime} \mathrm{N} \\
139^{\circ} 16^{\prime} 46.715^{\prime \prime} \mathrm{E}\end{array}$ & $\begin{array}{l}35^{\circ} 01^{\prime} 32.9933^{\circ} \mathrm{N} \\
139^{\circ} 29^{\prime} 08.094^{\prime \prime} \mathrm{E}\end{array}$ & $\begin{array}{c}2006-02 / 04 \\
0: 24: 27 \\
\end{array}$ & $1224-2377$ & 28.85 \\
\hline $\begin{array}{c}\text { SG06-KY-GA } \\
\text { R } \\
\end{array}$ & $\begin{array}{l}35^{\circ} 00^{\prime} 24.9299^{\prime} \mathrm{N} \\
139^{\circ} 26^{\prime} 46.648^{\prime \prime} \mathrm{E}\end{array}$ & $\begin{array}{l}35^{\circ} 06^{\prime} 28.880^{\prime \prime} \mathrm{N} \\
139^{\circ} 20^{\prime} 24.081^{\prime \prime} \mathrm{E}\end{array}$ & $\begin{array}{c}2006-02 / 04 \\
4: 43: 43 \\
\end{array}$ & $1002-1595$ & 14.85 \\
\hline SG06-KY- IA & $\begin{array}{c}35^{\circ} 08^{\prime} 58.413^{\prime \prime} \mathrm{N} \\
139^{\circ} 24^{\prime} 49.7333^{\prime \prime} \mathrm{E}\end{array}$ & $\begin{array}{l}35^{\circ} 02^{\prime} 54.402^{\prime \prime} \mathrm{N} \\
139^{\circ} 31^{\prime} 06.341 \text { ”E }\end{array}$ & $\begin{array}{l}2006-02 / 04 \\
7: 43: 17\end{array}$ & $1002-1591$ & 14.75 \\
\hline SG06-KY-J & $\begin{array}{l}35^{\circ} 03^{\prime} 55.132^{\prime \prime} \mathrm{N} \\
139^{\circ} 31^{\prime} 45.204^{\prime \prime} \mathrm{E}\end{array}$ & $\begin{array}{l}35^{\circ} 09^{\prime} 40.122^{\prime \prime} \mathrm{N} \\
139^{\circ} 25^{\prime} 45.518^{\prime \prime} \mathrm{E}\end{array}$ & $\begin{array}{c}2006-02 / 04 \\
10: 01: 20 \\
\end{array}$ & $1002-1562$ & 14.025 \\
\hline SG06-KY-JA & $\begin{array}{c}35^{\circ} 10^{\prime} 06.922^{\prime \prime} \mathrm{N} \\
139^{\circ} 27^{\prime} 05.055^{\prime} \mathrm{E}\end{array}$ & $\begin{array}{l}35^{\circ} 04^{\prime} 18.862^{\prime \prime} \mathrm{N} \\
139^{\circ} 33^{\prime} 07.926^{\prime} \mathrm{E}\end{array}$ & $\begin{array}{c}2006-02 / 04 \\
12: 11: 53 \\
\end{array}$ & $1026-1591$ & 14.15 \\
\hline SG06-KY-KA & $\begin{array}{l}35^{\circ} 05^{\prime} 40.5033^{\prime \prime} \mathrm{N} \\
139^{\circ} 34^{\prime} 18.841^{\prime \prime} \mathrm{E}\end{array}$ & $\begin{array}{ll}35^{\circ} & 12^{\prime} 39.329^{\prime} \mathrm{N} \\
139^{\circ} & 27^{\prime} 06.280^{\prime \prime} \mathrm{E}\end{array}$ & $\begin{array}{c}2006-02 / 04 \\
14: 32: 53 \\
\end{array}$ & $1002-1679$ & 16.95 \\
\hline SG06-KY-50 & $\begin{array}{c}35^{\circ} 12^{\prime} 21.061 ” \mathrm{~N} \\
139^{\circ} 26^{\prime} 29.174 ” \mathrm{E}\end{array}$ & $\begin{array}{l}35^{\circ} 02^{\prime} 59.213^{\prime \prime} \mathrm{N} \\
139^{\circ} 13^{\prime} 08.9744^{\prime \prime} \mathrm{E}\end{array}$ & $\begin{array}{c}2006-02 / 04 \\
17: 01: 02 \\
\end{array}$ & $1262-2328$ & 26.675 \\
\hline
\end{tabular}


Table 2: (Continued)

\begin{tabular}{|c|c|c|c|c|c|}
\hline D1 & $\begin{array}{c}34-58.2461^{\prime} \\
139-15.7883^{\prime}\end{array}$ & $\begin{array}{r}34-58.2626^{\prime} \mathrm{N} \\
139-13.7640^{\prime}\end{array}$ & $\begin{array}{c}2006 / 02 / 08 \\
23: 30: 06\end{array}$ & $1492-1912$ & 3.11 \\
\hline D 2 & $\begin{array}{r}34-58.3244^{\prime} \mathrm{N} \\
139-15.7608^{\prime}\end{array}$ & $\begin{array}{c}34-58.3083^{\prime} \mathrm{N} \\
139-13.7476^{\prime} \mathrm{E}\end{array}$ & $\begin{array}{c}2006 / 02 / 09 \\
0: 37: 25\end{array}$ & $2490-2903$ & 3.08 \\
\hline D3 & $\begin{array}{c}34-58.3878^{\prime} \mathrm{N} \\
139-15.7965^{\prime} \mathrm{E}\end{array}$ & $\begin{array}{c}34-58.3527^{\prime} \mathrm{N} \\
139-13.7448^{\prime} \mathrm{E}\end{array}$ & $\begin{array}{c}2006 / 02 / 09 \\
1: 43: 17 \\
\end{array}$ & $3466-3866$ & 3.14 \\
\hline D4 & $\begin{array}{c}34-58.4187^{\prime} \mathrm{N} \\
139-13.7997^{\prime} \mathrm{E} \\
\end{array}$ & $\begin{array}{c}34-58.4090 ' \mathrm{~N} \\
139-15.7965^{\prime} \mathrm{E} \\
\end{array}$ & $\begin{array}{c}2006 / 02 / 08 \\
22: 56: 51 \\
\end{array}$ & $1427-1491$ & 3.09 \\
\hline D5 & $\begin{array}{c}34-58.4587^{\prime} \mathrm{N} \\
139-13.7759^{\prime} \mathrm{E}\end{array}$ & $\begin{array}{c}34-58.4564^{\prime} \mathrm{N} \\
139-15.8084^{\prime} \mathrm{E}\end{array}$ & $\begin{array}{c}2006 / 02 / 09 \\
0: 04: 26 \\
\end{array}$ & $2001-2417$ & 3.11 \\
\hline D6 & $\begin{array}{c}34-58.5381^{\prime} \mathrm{N} \\
139-13.7842^{\prime} \mathrm{E}\end{array}$ & $\begin{array}{r}34-58.5134^{\prime} \mathrm{N} \\
139-15.8194^{\prime}\end{array}$ & $\begin{array}{c}2006 / 02 / 09 \\
1: 11: 03\end{array}$ & $2988-3404$ & 3.12 \\
\hline D7 & $\begin{array}{c}34-58.5651^{\prime} \mathrm{N} \\
139-15.6958^{\prime} \mathrm{E}\end{array}$ & $\begin{array}{c}34-58.5629^{\prime} \mathrm{N} \\
139-13.7686^{\prime} \mathrm{E}\end{array}$ & $\begin{array}{c}2006 / 02 / 08 \\
22: 25: 01\end{array}$ & $525-916$ & 2.95 \\
\hline D8 & $\begin{array}{c}34-58.6210^{\prime} \mathrm{N} \\
139-13.7833^{\prime} \mathrm{E}\end{array}$ & $\begin{array}{c}34-58.6107^{\prime} \mathrm{N} \\
139-16.0574^{\prime} \mathrm{E}\end{array}$ & $\begin{array}{c}2006 / 02 / 09 \\
2: 15: 55\end{array}$ & $3949-4393$ & 3.48 \\
\hline D9 & $\begin{array}{c}34-58.6702^{\prime} \mathrm{N} \\
139-15.8240^{\prime} \mathrm{E}\end{array}$ & $\begin{array}{r}34-58.6583^{\prime} \mathrm{N} \\
139-13.7238^{\prime} \mathrm{E}\end{array}$ & $\begin{array}{c}2006 / 02 / 09 \\
2: 53: 37 \\
\end{array}$ & $4508-4913$ & 3.21 \\
\hline D10 & $\begin{array}{c}34-58.7045^{\prime} \mathrm{N} \\
139-13.6899^{\prime} \mathrm{E}\end{array}$ & $\begin{array}{c}34-58.7128^{\prime} \mathrm{N} \\
139-15.8038^{\prime} \mathrm{E}\end{array}$ & $\begin{array}{c}2006 / 02 / 08 \\
21: 49: 47\end{array}$ & $2-438$ & 3.23 \\
\hline D21 & $\begin{array}{c}34-58.9435^{\prime} \mathrm{N} \\
139-14.5779^{\prime} \mathrm{E}\end{array}$ & $\begin{array}{c}34-57.8380^{\prime} \mathrm{N} \\
139-14.5862^{\prime} \mathrm{E}\end{array}$ & $\begin{array}{c}2006 / 02 / 09 \\
3: 37: 45\end{array}$ & $26-283$ & 2.06 \\
\hline D22 & $\begin{array}{c}34-59.1779^{\prime} \mathrm{N} \\
139-14.6475^{\prime} \mathrm{E}\end{array}$ & $\begin{array}{c}34-57.8181^{\prime} \mathrm{N} \\
139-14.6384^{\prime} \mathrm{E}\end{array}$ & $\begin{array}{c}2006 / 02 / 09 \\
5: 18: 41\end{array}$ & $1458-1771$ & 2.53 \\
\hline D23 & $\begin{array}{c}34-59.1877^{\prime} \mathrm{N} \\
139-14.6970^{\prime} \mathrm{E} \\
\end{array}$ & $\begin{array}{c}34-57.8183^{\prime} \mathrm{N} \\
139-14.6841^{\prime} \mathrm{E} \\
\end{array}$ & $\begin{array}{c}2006 / 02 / 09 \\
6: 09: 13 \\
\end{array}$ & $2204-2522$ & 2.55 \\
\hline D24 & $\begin{array}{c}34-59.10766^{\prime} \mathrm{N} \\
139-14.7409^{\prime} \mathrm{E}\end{array}$ & $\begin{array}{c}34-57.8444^{\prime} \mathrm{N} \\
139-14.7363^{\prime} \mathrm{E}\end{array}$ & $\begin{array}{c}2006 / 02 / 09 \\
6: 59: 39\end{array}$ & $2948-3249$ & 2.34 \\
\hline D25 & $\begin{array}{c}34-57.7677^{\prime} \mathrm{N} \\
139-14.7968^{\prime} \mathrm{E}\end{array}$ & $\begin{array}{c}34-59.1444 \text { ' N } \\
139-14.7812^{\prime} \mathrm{E}\end{array}$ & $\begin{array}{c}2006 / 02 / 09 \\
4: 00: 09 \\
\end{array}$ & 297-609 & 2.56 \\
\hline D26 & $\begin{array}{c}34-57.8110^{\prime} \mathrm{N} \\
139-14.8270^{\prime} \mathrm{E}\end{array}$ & $\begin{array}{c}34-59.1339^{\prime} \mathrm{N} \\
139-14.8334^{\prime} \mathrm{E}\end{array}$ & $\begin{array}{c}2006 / 02 / 09 \\
4: 53: 41\end{array}$ & $1089-1384$ & 2.46 \\
\hline D27 & $\begin{array}{c}34-57.9149^{\prime} \mathrm{N} \\
139-14.8966^{\prime} \mathrm{E} \\
\end{array}$ & $\begin{array}{c}34-59.1410^{\prime} \mathrm{N} \\
139-14.8846^{\prime} \mathrm{E} \\
\end{array}$ & $\begin{array}{c}2006 / 02 / 09 \\
5: 45: 46 \\
\end{array}$ & $1858-2138$ & 2.28 \\
\hline D28 & $\begin{array}{c}34-57.89366^{\prime} \mathrm{N} \\
139-14.9277^{\prime} \mathrm{E} \\
\end{array}$ & $\begin{array}{c}34-59.1371^{\prime} \mathrm{N} \\
139-14.9387^{\prime} \mathrm{E}\end{array}$ & $\begin{array}{c}2006 / 02 / 09 \\
6: 35: 48 \\
\end{array}$ & $2596-2874$ & 2.31 \\
\hline D29 & $\begin{array}{c}34-59.1557^{\prime} \mathrm{N} \\
139-14.9799^{\prime} \mathrm{E}\end{array}$ & $\begin{array}{c}34-57.8121^{\prime} \mathrm{N} \\
139-14.9789^{\prime} \mathrm{E}\end{array}$ & $\begin{array}{c}2006 / 02 / 09 \\
4: 26: 22\end{array}$ & $685-1001$ & 2.50 \\
\hline D30 & $\begin{array}{c}34-57.8124{ }^{\prime} \mathrm{N} \\
139-15.0449^{\prime} \mathrm{E} \\
\end{array}$ & $\begin{array}{c}34-59.1129^{\prime} \mathrm{N} \\
139-15.0339^{\prime} \mathrm{E}\end{array}$ & $\begin{array}{c}2006 / 02 / 09 \\
7: 25: 35 \\
\end{array}$ & $3333-3649$ & 2.45 \\
\hline
\end{tabular}


Table 2: (Continued)

\begin{tabular}{|c|c|c|c|c|c|}
\hline 17 & $\begin{array}{c}34-47^{\prime} 46.429 \mathrm{~N} \\
139-11^{\prime} 52.284 \mathrm{E}\end{array}$ & $\begin{array}{l}35-15^{\prime} 22.898 \mathrm{~N} \\
139-21^{\prime} 07.166 \mathrm{E}\end{array}$ & $\begin{array}{c}2006 / 02 / 05 \\
5: 14: 01\end{array}$ & $1086-3204$ & 53.0 \\
\hline 18 & $\begin{array}{l}35-13^{\prime} 29.254 \mathrm{~N} \\
139-17^{\prime} 59.573 \mathrm{E}\end{array}$ & $\begin{array}{c}34-48^{\prime} 37.233 \mathrm{~N} \\
139-07^{\prime} 50.903 \mathrm{E}\end{array}$ & $\begin{array}{c}2006 / 02 / 05 \\
13: 00: 39\end{array}$ & $1002-2942$ & 48.5 \\
\hline 5 & $\begin{array}{c}34-58^{\prime} 29.653 \mathrm{~N} \\
139-12^{\prime} 46.123 \mathrm{E}\end{array}$ & $\begin{array}{l}35-02^{\prime} 49.810 \mathrm{~N} \\
139-14 ' 34.520 \mathrm{E}\end{array}$ & $\begin{array}{c}2006 / 02 / 05 \\
21: 53: 06\end{array}$ & $1002-1341$ & 8.5 \\
\hline 10 & $\begin{array}{l}35-02^{\prime} 17.281 \mathrm{~N} \\
139-14 ' 59.506 \mathrm{E}\end{array}$ & $\begin{array}{c}34-57^{\prime} 56.762 \quad \mathrm{~N} \\
139-13^{\prime} 09.400 \mathrm{E}\end{array}$ & $\begin{array}{c}2006 / 02 / 05 \\
23: 14: 27\end{array}$ & $1002-1342$ & 8.53 \\
\hline 12 & $\begin{array}{c}34-58^{\prime} 12.690 \mathrm{~N} \\
139-13^{\prime} 48.547 \mathrm{E}\end{array}$ & $\begin{array}{l}35-02^{\prime} 32.953 \mathrm{~N} \\
139-15^{\prime} 36.595 \mathrm{E}\end{array}$ & $\begin{array}{c}2006 / 02 / 06 \\
0: 34: 46\end{array}$ & $1002-1341$ & 8.5 \\
\hline 14 & $\begin{array}{l}35-02^{\prime} 01.570 \mathrm{~N} \\
139-15^{\prime} 51.418 \mathrm{E}\end{array}$ & $\begin{array}{l}34-57^{\prime} 41.124 \mathrm{~N} \\
139-14^{\prime} 04.102 \mathrm{E}\end{array}$ & $\begin{array}{c}2006 / 02 / 06 \\
1: 52: 11\end{array}$ & $1003-1342$ & 8.5 \\
\hline 11 & $\begin{array}{c}34-58^{\prime} 17.063 \mathrm{~N} \\
139-13^{\prime} 32.982 \mathrm{E}\end{array}$ & $\begin{array}{l}35-02^{\prime} 37.673 \mathrm{~N} \\
139-15^{\prime} 22.755 \mathrm{E}\end{array}$ & $\begin{array}{c}2006 / 02 / 06 \\
3: 13: 49\end{array}$ & $1002-1342$ & 8.53 \\
\hline 9 & $\begin{array}{l}35-01 ' 59.548 \mathrm{~N} \\
139-16^{\prime} 00.264 \mathrm{E}\end{array}$ & $\begin{array}{c}34-57^{\prime} 39.120 \mathrm{~N} \\
139-14^{\prime} 12.857 \mathrm{E}\end{array}$ & $\begin{array}{c}2006 / 02 / 06 \\
4: 39: 42\end{array}$ & $10002-1341$ & 8.5 \\
\hline 8 & $\begin{array}{c}34-58^{\prime} 23.618 \mathrm{~N} \\
139-13^{\prime} 07.368 \mathrm{E}\end{array}$ & $\begin{array}{l}35-02^{\prime} 44.803 \mathrm{~N} \\
139-14^{\prime} 55.104 \mathrm{E}\end{array}$ & $\begin{array}{c}2006 / 02 / 06 \\
6: 03: 39 \\
\end{array}$ & $1002-1342$ & 8.53 \\
\hline 13 & $\begin{array}{c}35-01 ' 15.981 \mathrm{~N} \\
139-15 ' 22.783 \mathrm{E}\end{array}$ & $\begin{array}{c}34-57^{\prime} 44.120 \mathrm{~N} \\
139-13^{\prime} 54.834 \mathrm{E}\end{array}$ & $\begin{array}{c}2006 / 02 / 06 \\
7: 41: 30\end{array}$ & $1066-1342$ & 6.93 \\
\hline 7 & $\begin{array}{c}34-58^{\prime} 25.734 \mathrm{~N} \\
139-12^{\prime} 59.903 \mathrm{E}\end{array}$ & $\begin{array}{l}35-02^{\prime} 46.736 \mathrm{~N} \\
139-14^{\prime} 48.320 \mathrm{E}\end{array}$ & $\begin{array}{c}2006 / 02 / 06 \\
8: 55: 13\end{array}$ & $1002-1342$ & 8.53 \\
\hline 15 & $\begin{array}{c}35-01 ' 59.898 \mathrm{~N} \\
139-15 ' 56.661 \mathrm{E}\end{array}$ & $\begin{array}{c}34-57^{\prime} 40.522 \mathrm{~N} \\
139-14^{\prime} 08.560 \mathrm{E}\end{array}$ & $\begin{array}{c}2006 / 02 / 06 \\
10: 24: 25\end{array}$ & $1003-1341$ & 8.48 \\
\hline 16 & $\begin{array}{l}34-57^{\prime} 59.798 \mathrm{~N} \\
139-14^{\prime} 32.396 \mathrm{E}\end{array}$ & $\begin{array}{l}35-02^{\prime} 19.896 \mathrm{~N} \\
139-16^{\prime} 20.951 \mathrm{E}\end{array}$ & $\begin{array}{c}2006 / 02 / 06 \\
11: 57: 52\end{array}$ & $1002-1341$ & 8.5 \\
\hline 6 & $\begin{array}{l}35-02 ' 24.468 \mathrm{~N} \\
139-14 ' 31.477 \mathrm{E}\end{array}$ & $\begin{array}{c}34-58^{\prime} 04.229 \mathrm{~N} \\
139-12^{\prime} 43.385 \mathrm{E}\end{array}$ & $\begin{array}{c}2006 / 02 / 06 \\
13: 29: 32\end{array}$ & $1002-1341$ & 8.5 \\
\hline V & $\begin{array}{c}34-59^{\prime} 34.456 \mathrm{~N} \\
139-10^{\prime} 18.667 \mathrm{E}\end{array}$ & $\begin{array}{l}34-57^{\prime} 25.642 \mathrm{~N} \\
139-18^{\prime} 00.737 \mathrm{E}\end{array}$ & $\begin{array}{c}2006 / 02 / 06 \\
15: 26: 30\end{array}$ & $1020-1515$ & 12.4 \\
\hline $\mathrm{T}$ & $\begin{array}{c}34-57^{\prime} 57.781 \mathrm{~N} \\
139-17^{\prime} 35.520 \mathrm{E}\end{array}$ & $\begin{array}{l}35-00^{\prime} 10.781 \mathrm{~N} \\
139-09^{\prime} 42.687 \mathrm{E}\end{array}$ & $\begin{array}{c}2006 / 02 / 06 \\
17: 16: 52\end{array}$ & $1007-1514$ & 12.7 \\
\hline $\mathrm{O}$ & $\begin{array}{c}35-00^{\prime} 40.582 \mathrm{~N} \\
139-11^{\prime} 20.455 \mathrm{E}\end{array}$ & $\begin{array}{c}34-58^{\prime} 40.096 \mathrm{~N} \\
139-18^{\prime} 29.647 \mathrm{E}\end{array}$ & $\begin{array}{c}2006 / 02 / 06 \\
19: 10: 10\end{array}$ & $1053-1513$ & 11.53 \\
\hline $\mathrm{U} 2$ & $\begin{array}{c}34-57^{\prime} 50.254 \mathrm{~N} \\
139-16^{\prime} 57.482 \mathrm{E}\end{array}$ & $\begin{array}{c}34-59 ' 53.418 \mathrm{~N} \\
139-09^{\prime} 35.940 \mathrm{E}\end{array}$ & $\begin{array}{c}2006 / 02 / 06 \\
21: 08: 02\end{array}$ & $141-1514$ & 11.85 \\
\hline $\mathrm{N}$ & $\begin{array}{l}35-00^{\prime} 42.965 \mathrm{~N} \\
139-11^{\prime} 31.887 \mathrm{E}\end{array}$ & $\begin{array}{c}34-58^{\prime} 46.121 \mathrm{~N} \\
139-18^{\prime} 32.130 \mathrm{E}\end{array}$ & $\begin{array}{c}2006 / 02 / 06 \\
23: 07: 57\end{array}$ & $1063-1513$ & 11.28 \\
\hline $\mathrm{U}$ & $\begin{array}{c}34-57^{\prime} 49.349 \mathrm{~N} \\
139-17^{\prime} 22.987 \mathrm{E}\end{array}$ & $\begin{array}{l}34-59^{\prime} 59.704 \mathrm{~N} \\
139-09^{\prime} 38.430 \mathrm{E}\end{array}$ & $\begin{array}{c}2006 / 02 / 07 \\
0: 57: 32\end{array}$ & $1016-1514$ & 12.48 \\
\hline
\end{tabular}


Table 3: MCS line information of Leg 2 for the western Sagami Bay.

\begin{tabular}{|c|c|}
\hline Date & Remarks \\
\hline February 5 & $\begin{array}{l}\text { Departure from Ito port } \\
\text { Adjustment of investment equipments and start airguns shooting }\end{array}$ \\
\hline February 6 & $\begin{array}{l}\text { Continue MCS survey } \\
\text { Recovery airguns and streamer due to bad weather }\end{array}$ \\
\hline February 7 & $\begin{array}{l}\text { Deploy airguns and streamer } \\
\text { Restart MCS survey } \\
\text { Recovery airguns and streamer due to Streamer's bird trouble }\end{array}$ \\
\hline February 8 & $\begin{array}{l}\text { Deploy airguns and streamer } \\
\text { Restart MCS survey }\end{array}$ \\
\hline February 9 & $\begin{array}{l}\text { Continue MCS survey } \\
\text { Recovery airguns and streamer due to bad weather }\end{array}$ \\
\hline February 10 & $\begin{array}{l}\text { Deploy airguns and streamer } \\
\text { Restart MCS survey }\end{array}$ \\
\hline February 11 & $\begin{array}{l}\text { Continue MCS survey } \\
\text { Recovery airguns and streamer } \\
\text { Transit to JAMSTEC } \\
\text { Arrival at JAMSTEC end of KY0601 }\end{array}$ \\
\hline
\end{tabular}

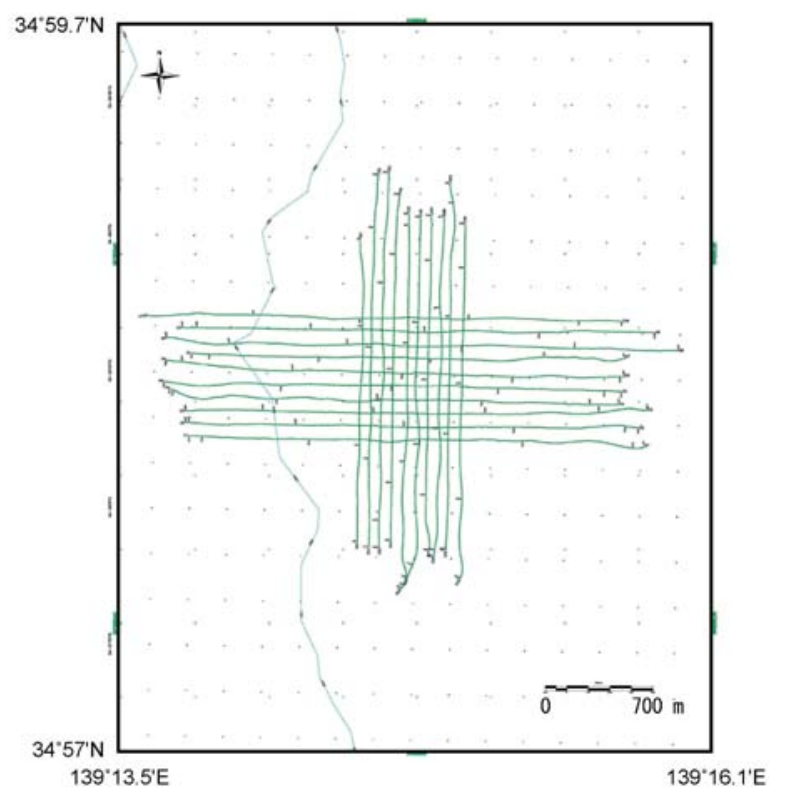

Figure 2: SCS survey line around the Hatsushima cold seep communities.

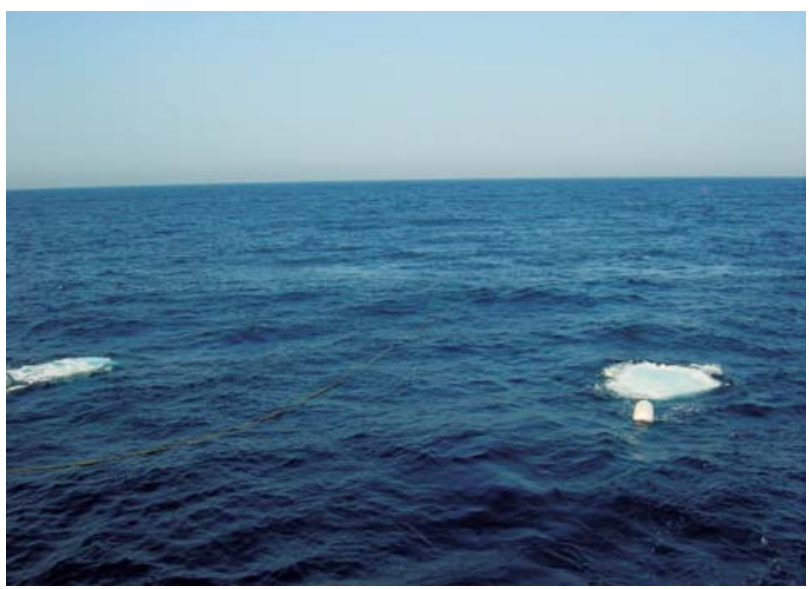

Figure 3: Photograph of double shooting GI gun system ready for launching onboard Kaiyo. 

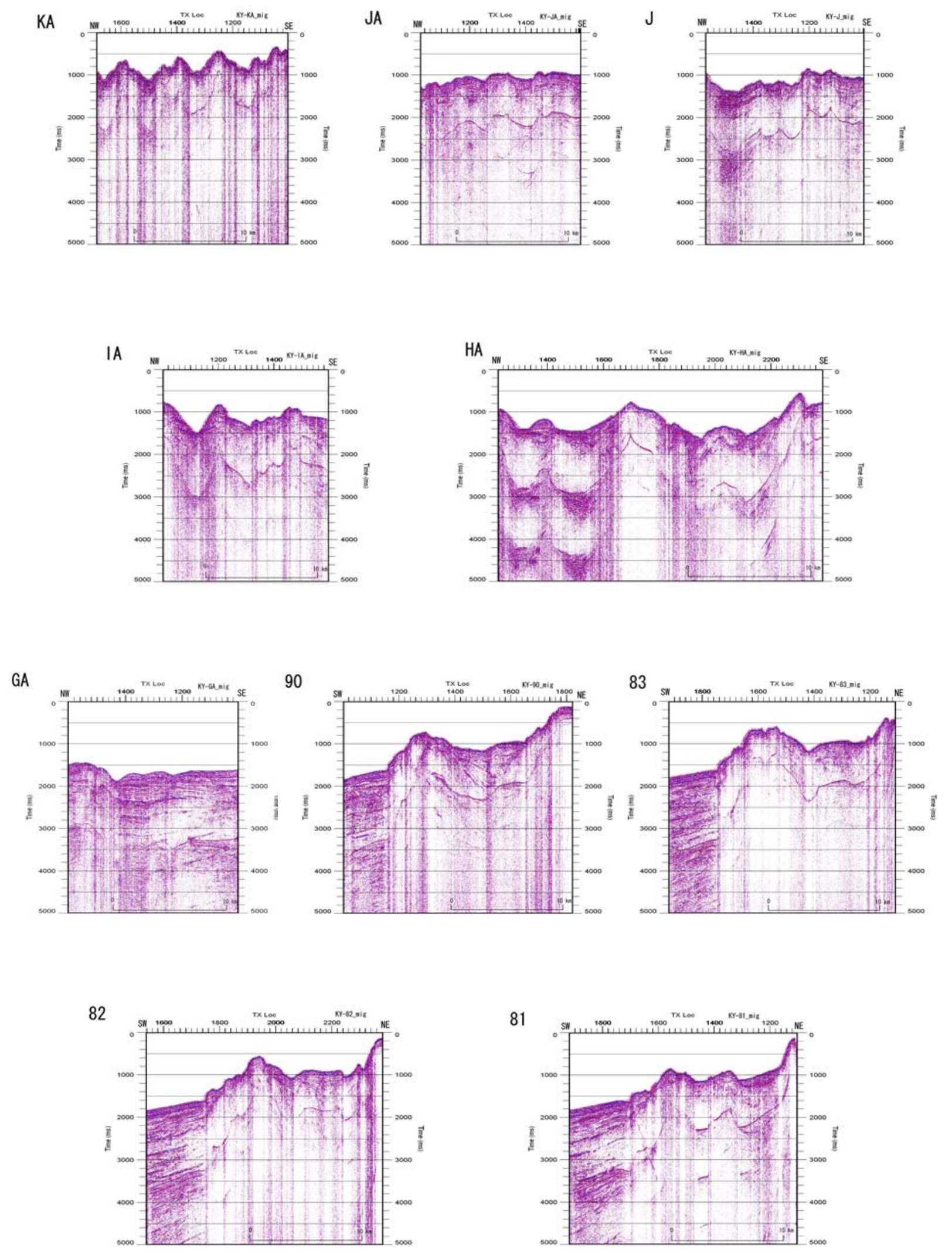

Figure 4: MCS profiles in the eastern Sagami Bay area (Leg1). Vertical axis is a two-way travel time in second. 
80

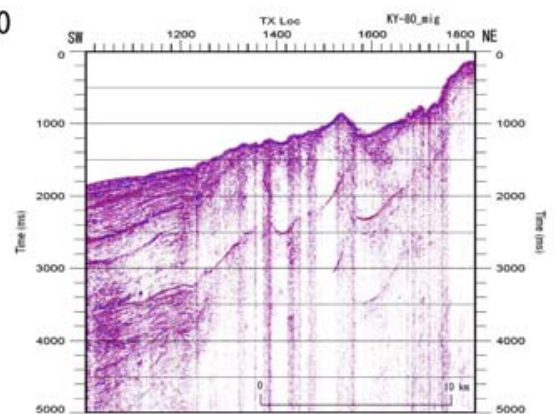

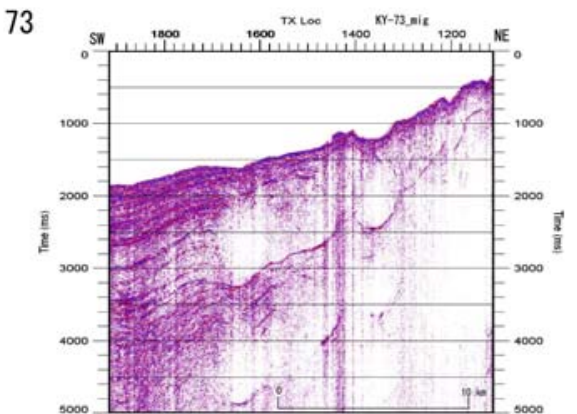
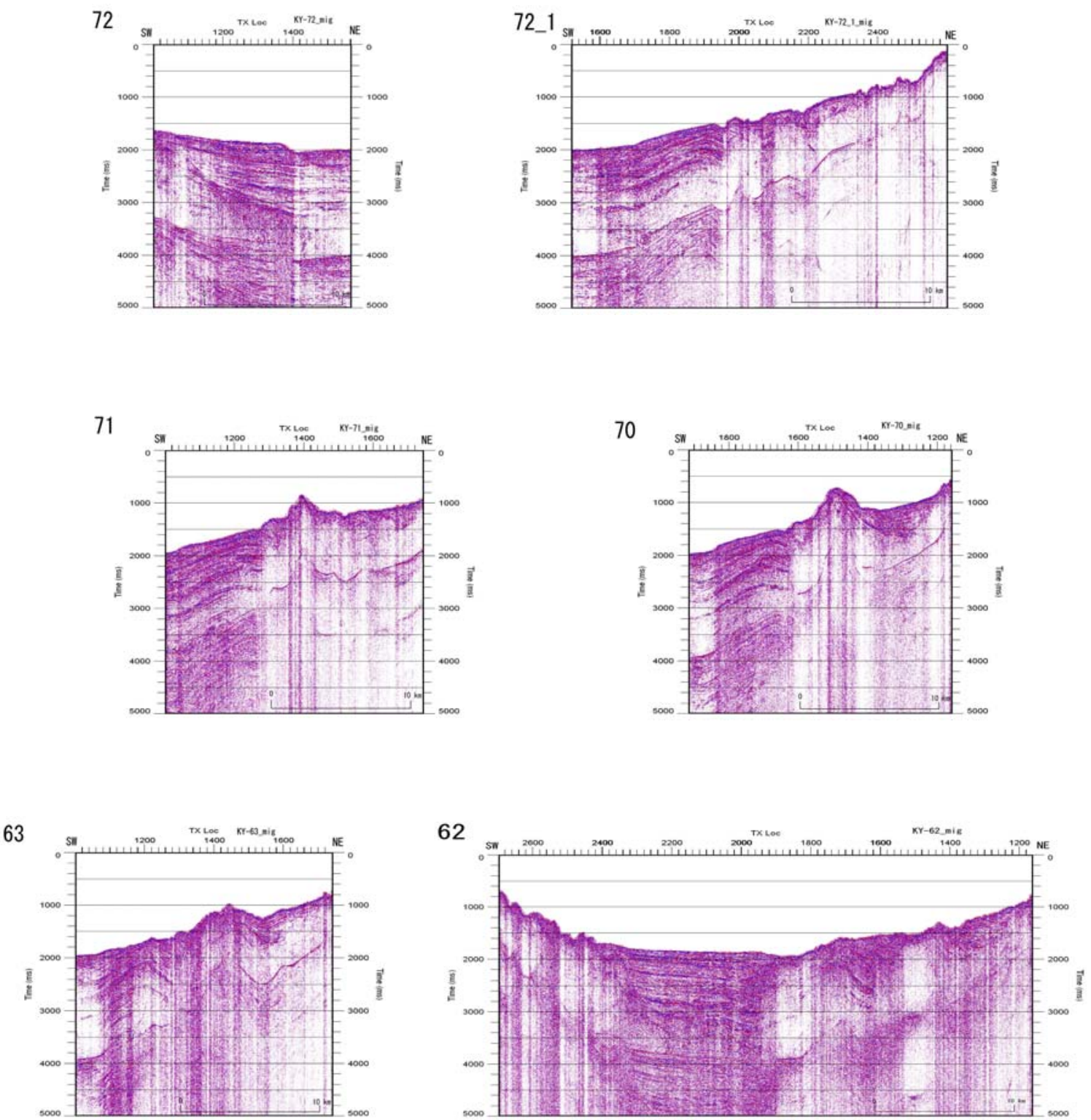

Figure 4: (Continued) 

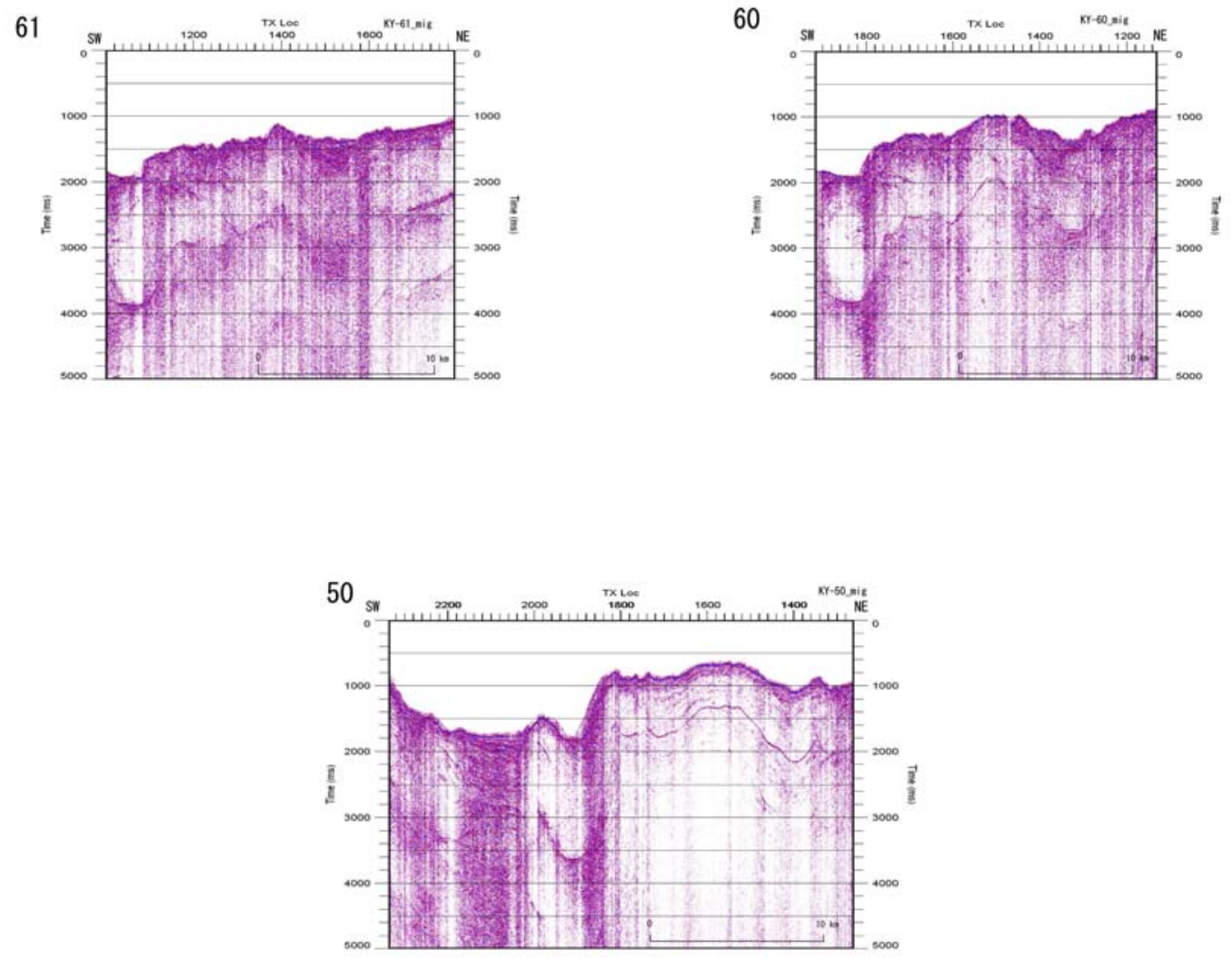

Figure 4: (Continued) 

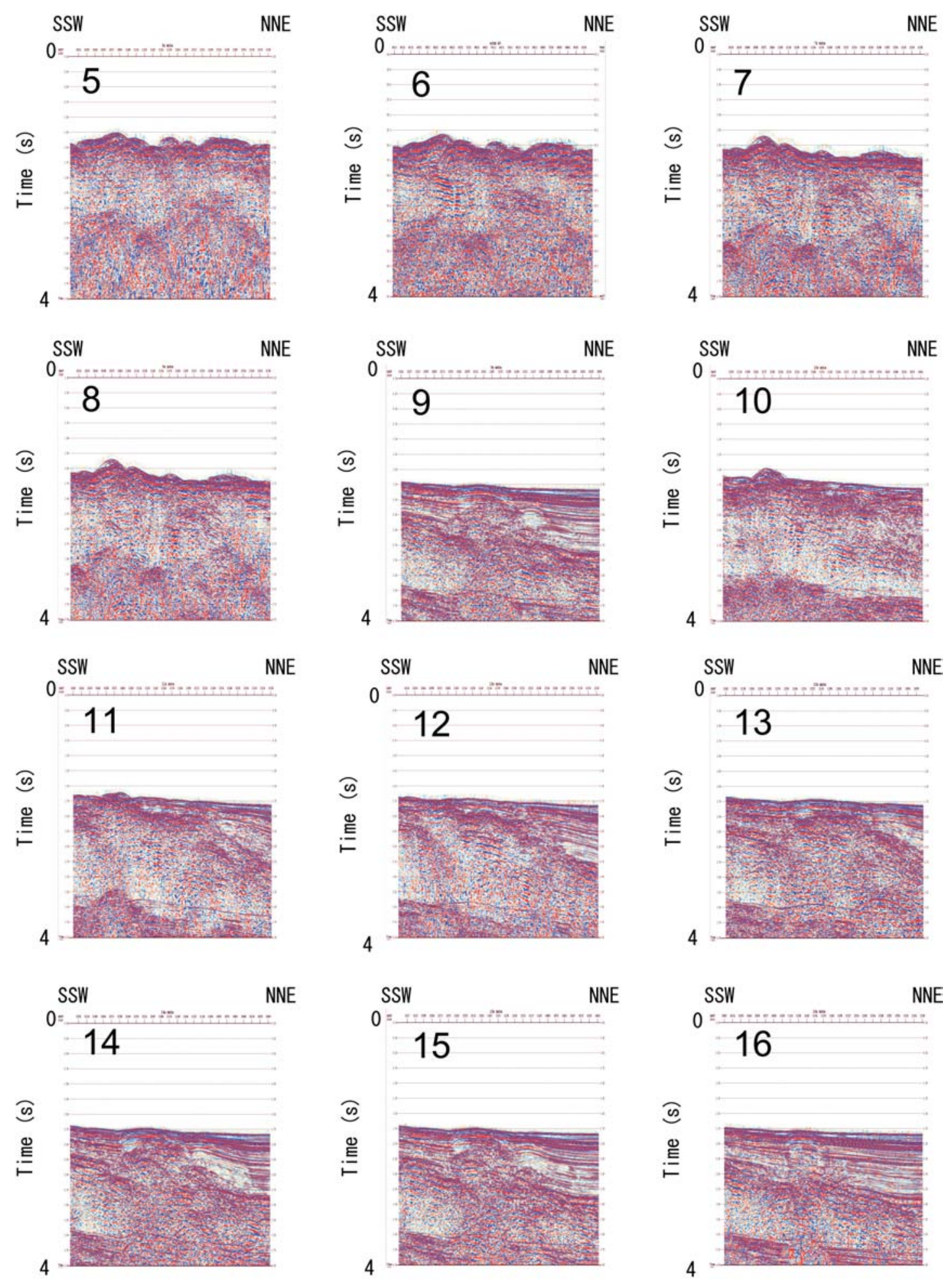

Figure 5: MCS profiles in the western Sagami Bay area (Leg2). Vertical axis is a two-way travel time in second. 

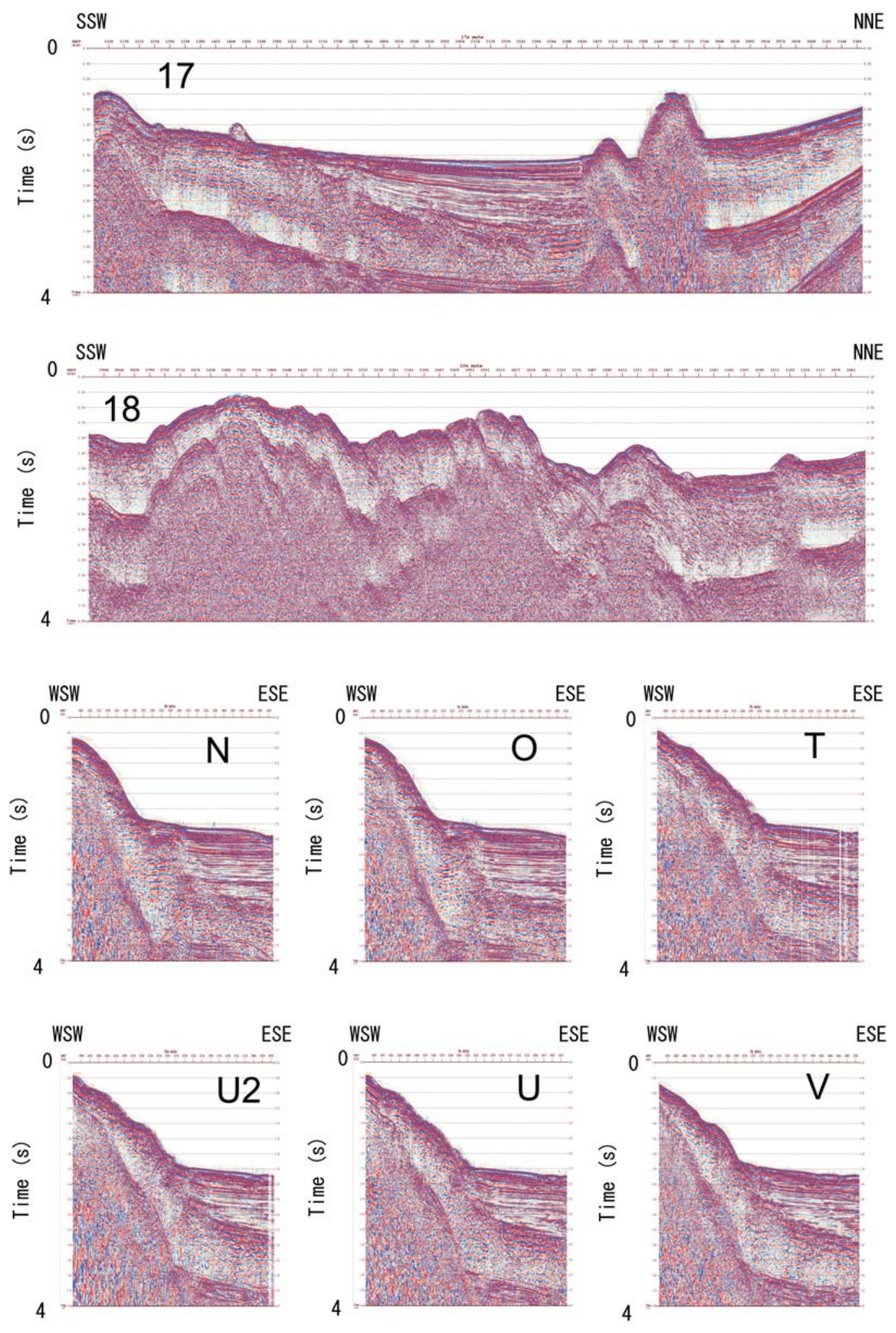

Figure 5: (Continued) 

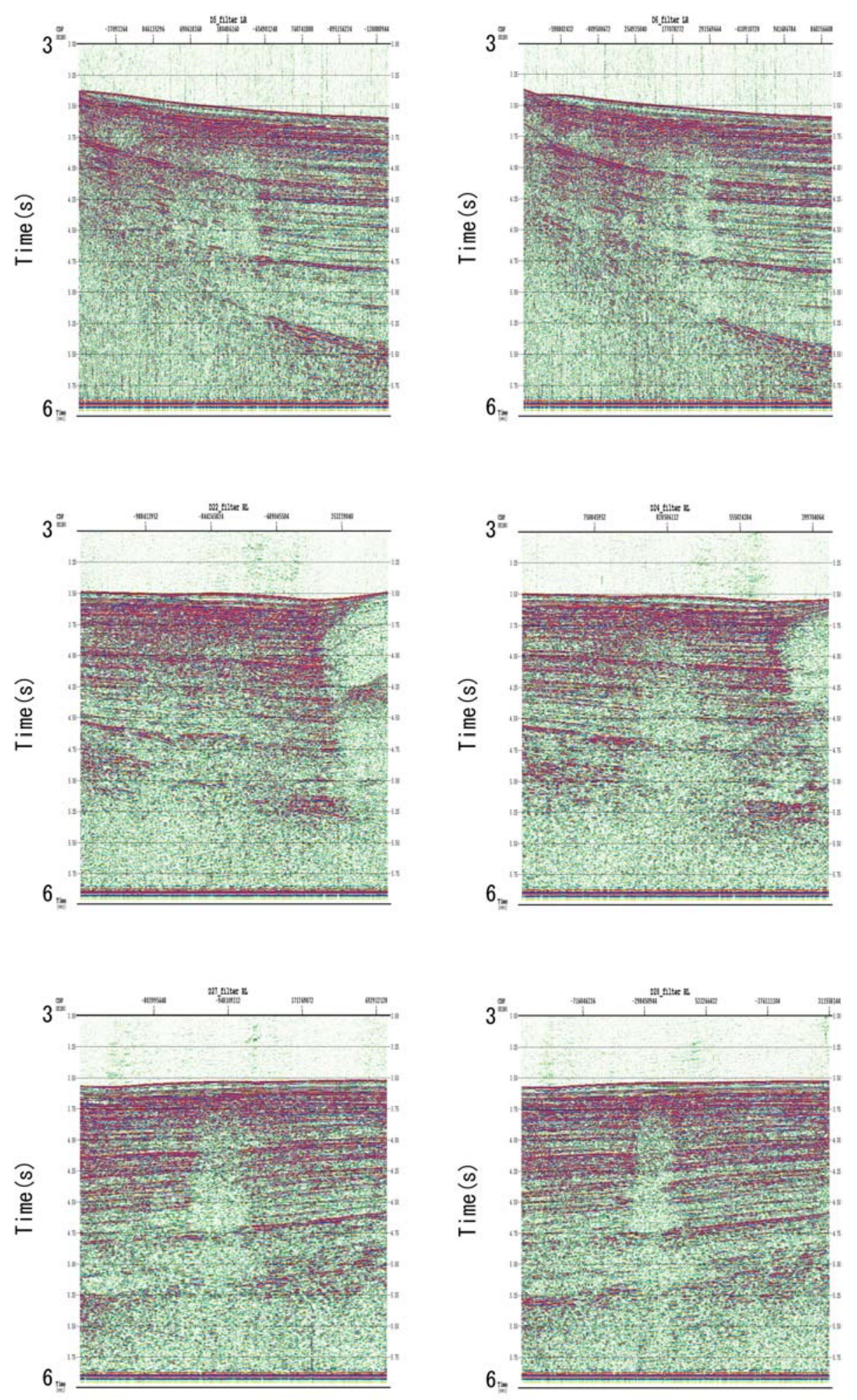

Figure 6: Example of SCS profiles around the Hatsushima site Vertical axis is a two-way travel time in second. 